Meta

Journal des traducteurs

Translators' Journal

\title{
La récré du traducteur
}

\section{Alain Van Crugten}

Volume 34, numéro 1, mars 1989

Humour et traduction

Humour and Translation

URI : https://id.erudit.org/iderudit/002978ar

DOI : https://doi.org/10.7202/002978ar

Aller au sommaire du numéro

Éditeur(s)

Les Presses de l'Université de Montréal

ISSN

0026-0452 (imprimé)

1492-1421 (numérique)

Découvrir la revue

Citer cet article

Van Crugten, A. (1989). La récré du traducteur. Meta, 34(1), 26-32.

https://doi.org/10.7202/002978ar

Ce document est protégé par la loi sur le droit d'auteur. L'utilisation des services d'Érudit (y compris la reproduction) est assujettie à sa politique d'utilisation que vous pouvez consulter en ligne.

https://apropos.erudit.org/fr/usagers/politique-dutilisation/
Cet article est diffusé et préservé par Érudit.

Érudit est un consortium interuniversitaire sans but lucratif composé de l'Université de Montréal, l'Université Laval et l'Université du Québec à Montréal. Il a pour mission la promotion et la valorisation de la recherche. https://www.erudit.org/fr/ 


\section{LA RÉCRÉ DU TRADUCTEUR}

Alain VAN CRUGTEN

Se souvient-on encore de ce chef-d'œuvre de la littérature française, paru en plusieurs séries entre 1907 et 1925 sous le titre A la manière de...? Paul Reboux et Charles Muller y avaient réuni les plus hilarants des pastiches littéraires. L'un des morceaux les plus réussis est un pastiche de Tolstoï et les meilleurs en général sont ceux des textes russes traduits en français : c'est dire qu'on y parodie non seulement Tolstoï, mais aussi les traducteurs. On y voit comment, à cette époque (la chose est évidemment impensable à présent, n'est-ce pas?), les traducteurs résolvaient leurs problèmes au moyen de notes explicatives :

À partir de ce jour, Ivan Labibine changea de $\operatorname{krokno}^{1}$ (...)

Enfin il alla faire un mychew ${ }^{2}$ dans un des massifs de la Perspective Newsky (...)

Il venait de remplir cet acte salutaire quand il fut accosté par une prostituée.

«Dis-moi quoi, joli bronskoï 3 , viens chez moi, il y a du vodnia ${ }^{4}$ ».

Ivan Labibine Ossouzoff prit les mains de cette malheureuse et les embrassa. Mais elle se recula avec frayeur. (...)

La fille riposta :

«Donne-moi plutôt trois kopecks ${ }^{5}$ pour acheter du kwass 6 .

- Je te donnerai gratuitement des meurbacks ${ }^{7}$ », poursuivit affectueusement Ivan Labibine.

Ces extraits parodiques pourraient faire croire à la théorie «extrémiste» selon laquelle rien n'est traduisible. Il n'en est rien, la traduction existe, je l'ai rencontrée. J'ai même vu des traducteurs heureux.

Mais il est un fait indiscutable et indiscuté : dans toute traduction, il y a une perte de sens. Or, il apparaît que s'il est un domaine où cette perte est importante, c'est bien celui du registre humoristique. L'humour étant une de ces choses presque indéfinissables et inanalysables, il est souvent malaisé de le transmettre dans une traduction qui est, en réalité, une explication.

Heureusement pour les auteurs de textes humoristiques, les traducteurs d'aujourd'hui ont plus de conscience professionnelle que les modèles de Reboux et Muller et s'efforcent de trouver, sinon des traductions, du moins des équivalences satisfaisantes pour les passages qui leur offrent des difficultés.

Le hasard - ou peut-être n'est-ce pas tout a fait le hasard - a voulu que je traduise des auteurs qui, tout en disant des choses fort sérieuses, manifestent un sens ludique de la langue. Il s'agit notamment du Flamand Hugo Claus (Le Chagrin des Belges), des Polonais Stanislaw Ignacy Witkiewicz et Marian Pankowski (thêatre et romans) et de l'Anglais Robert Nye (Faust). Ce sont des écrivains qui aiment le mot, qui le caressent, le retournent, le manipulent avec amour, mais surtout qui traitent le sens et le son de la parole comme un objet de jeu, peut-être parce que, dans une certaine mesure, ils ont gardé l'esprit d'enfance sous la carapace de l'adulte et qu'ils considèrent l'existence comme une tragédie dont il vaut mieux rire. Ce sont aussi, chacun à sa manière, des virtuoses, des jongleurs, auxquels une riche connaissance de leur langue et des langues 
étrangères permet des acrobaties verbales qui font tout d'abord bien rire le traducteur, dont la gaieté se transforme vite en angoisse lorsqu'il se met en devoir de reproduire cet humour en français.

Il est bien évident que les pertes de sens sont inévitables lorsque de tels auteurs lâchent la bride à leur démon du grotesque, de l'humour, de la dérision. Dans ma pratique, je me suis donc parfois trouvé devant des obstacles insurmontables (pour moi); devant d'autres que j'ai hypocritement contournés; d'autres encore que j'ai franchis vaille que vaille et certains, hélas les moins nombreux, que j'ai sautés avec le sentiment satisfait d'avoir découvert le moyen de perdre un minimum de connotations humoristiques.

Voyons d'abord ce qui me désole, les échecs, les traductions impossibles.

Tiré du roman Faust de Robert Nye:

The Wartburg. That castle near Eisenach. Like a wart it is, too. A brown papillary excrescence on top of a hill ${ }^{8}$.

Le Wartburg. Le château près d'Eisenach. Comme une verrue. Une excroissance papillaire brune au sommet d'une colline?.

Il y a là un jeu de mots bilingue, allemand-anglais, portant sur l'homonymie de ces deux mots Wart / wart. Je ne pouvais que traduire littéralement, perdant ainsi l'allusion humoristique.

Dans le même roman, le narrateur, à propos de Johann Faust et de son pacte, ne perd aucune occasion d'affirmer qu'il ne croit ni à Dieu ni à diable :

Faustus will be saved. (Damned by his reckoning.)

There'll be high jinks in heaven

God will be rubbing his hands

Well, God won't be rubbing his hands.

Why not?

Because he hasn't got hands to rub.

Look, God, no hands.

Look, Hans, no God. (p. 94)

Ici encore je suis échec et mat. J'ai dû laisser tomber la dernière phrase et me borner a écrire :

Pas de mains, pas de Dieu.

Évidemment, je voyais l'allusion à la blague enfantine bien connue: «Regarde, Maman, sans les mains !» Mais on ne pouvait pas dire «Regarde, Dieu, sans les mains» et surtout il était impossible de reproduire le jeu phonétique portant sur le diminutif de Johannes, Hans, et l'anglais hands.

Une autre difficulté, illustrée également par des exemples tirés de Nye: la complexité que provoque chez le lecteur une référence connue lui arrache toujours un sourire. Cette connaissance de la référence permet parfois au texte original une concision que ne peut atteindre la traduction.

Chris Wagner, le narrateur, disciple de Faust, possède un singe nommé Ackercocke qu'il déteste. Il dit à un certain moment :

At least, Ackercocke is sleeping. Small mercies. (p. 60) 
Ce qui est une allusion à l'expression «thank God for small mercies». Je n'ai pu traduire qu'en allongeant :

Dieu soit loué pour nos petits bonheurs. (p. 87)

Autre clin d'œil au lecteur anglais: à Wagner qui l'interroge sur la grande tâche secrète qu'il doit accomplir à Rome, Faust répond laconiquement: «Murder», he said; ce qui rappelle un titre célèbre d'Agatha Christie: Murder, she said.

Il y a tout de même quelques moments où le traducteur s'octroie le droit de s'amuser un peu, parce que le contexte le permet, parce qu'il y a moyen, tout en s'écartant de la traduction littérale, de rester dans le registre, avec un minimum de déperdition de connotations. Toujours dans le même roman (très paillard) de Robert Nye, le narrateur se présente:

I'm Christopher Wagner. Never mind about my parents. They didn't give two fucks about me. Just the one fuck. Ha ha. (p. 9)

Je suis Christophe Wagner. Passons sur mes parents. Ils se foutaient de moi comme de leur première culotte. Tout juste s'ils ont pris la peine de l'ôter pour me faire. (p. 26)

Une fois de plus, je n'ai pas réussi à conserver la concision de l'original.

Ce type d'adaptation peut en général être réalisé, au prix d'un effort de réflexion et d'invention, lorsque l'auteur original se situe dans le registre parodique: puisqu'il effectue lui-même une sorte d'imitation comique, on se sent en droit de procéder de même avec son texte. J'avoue le plaisir que je prends à récrire à ma façon les poèmes ou chansonnettes grotesques dont Marian Pankowski parsème ses pièces de théâtre.

Dans la pièce pseudo-historique la Mort d' un bas blanc, les armées du traître lituanien Kiejstut assiègent en chantant le château de Jagellon, grand-duc de Lituanie et roi de Pologne:

WóJ I przytupując do rytmu, ale wylqcznie prawq nogq, spiewa nawet dośc czystym glosem

Tam gdzie boje-tam woje!

Hej! tra-la-laaa!

Niesiem slome specjalng,

prawie samozapalng,

Jagie lle zadymim, ha-ha-haaa!

Wykurzymy w try migi

lysy leb Pandziadygi;

ze smolaków dymisty jest dym!

Pierdnie dwakroc i kapnie

i dziadyga zakrztusi sie nim! $1^{\text {er }}$ Guerrier, marquant de son pas le rythme de la chanson, mais exclusivement du pied droit, et chantant d'une voix pas tellement fausse. -

Fonçons dans la bataille, Apportons de la paille Et des fagots d'érable Presque auto-inflammables !

Nous allons enfumer Jagellon, ce fumier! Ha-ha-ha!

Nous grill'rons en cinq sec Le crân' de ce vieux mec ; Il sera rondement Fumé comme un hareng.

Au fond de son donjon Crèv'ra le vieux cochon Et de la queue au groin Il sera cuit à point. 10 
Peu après, dans son château en flammes, Jagellon chante, en un ralenti mélancolique un air polonais connu, en changeant les paroles : «Quoique la tempête gronde autour de nous...» devient «Quoique la fumée nous prenne à la gorge...». Dans ma version française, il chante la jadis célèbre chanson militariste de Déroulède «Le clairon» («L'air est pur, la route est large — Le clairon sonne la charge», etc.) Mais les paroles sont également modifiées :

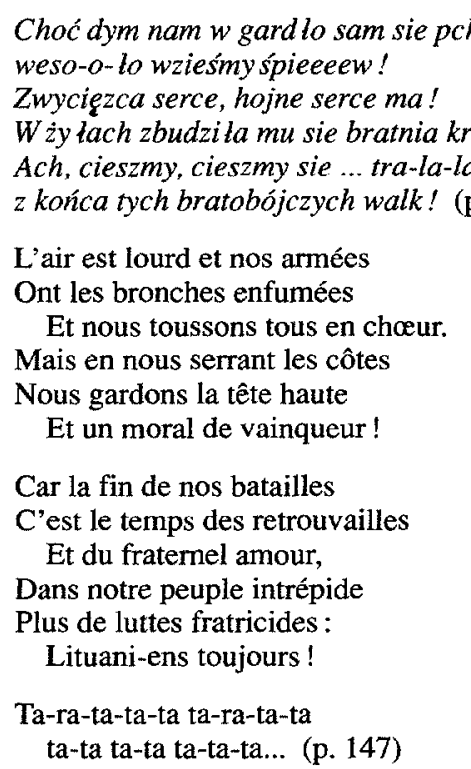

Ensuite Kiejstut est tué et son fils entonne un chant de vengeance. Cette vengeance étant, dans l'original, de type sado-scatologique, cela devient en français:

Sznurem, jak wieprza! Pod mury go wlec.

Kqsing pokrzywq po slepiach go siec!

Az rylem charczgcym zawyje do gwiazd!

Odrzé́ ze skóry $i$ w rany mu szczać!

I w gnoju zbrodnika ponurzyć po pas!

Aaaaa... (p. 136)

Qu'on entaille, qu'on taillade -

balafres, estafilades -

que l'on fende, qu'on pourfende,

qu'on tronçonne, qu'on moignonne,

qu'on mutile et qu'on ampute,

que l'on châtre et qu'on charcute

cette abominable brute,

cet immonde fils de pute!

Qu'il gueule «mea culpa!!»

l'assassin de mon papa - aaah...11 (p. 149)

Dans le cas des chansons de Pankowski, je me trouvais dans la situation privilégiée du traducteur auquel l'auteur laisse toute liberté ; je devais parodier poème ou chanson en gardant le sens général avec une forme au choix. Mais il arrive que la forme soit imposée 
avec plus ou moins de rigueur par le fait que l'auteur a voulu faire un jeu de sonorités. Il faut alors tenter de reproduire allitérations, assonances, paronomases, etc. Claus ${ }^{12}$.

Voici quelques exemples tirés du Chagrin des Belges, le chef-d'œuvre de Hugo

Le héros, Louis, dix ans, fait subir à un petit copain une torture humiliante parce qu'il se juge trahi par lui. L'enfant est plongé la tête la première dans un grand panier et subit des sévices. Le chapitre s'intitule en néerlandais «De mande va de schande», soit, littéralement, «Le panier de la honte» (p. 271). Mais il fallait tenir compte du fait que Louis, apprenti poète, se délecte de la formule rimée et chantonne la petite phrase comme une litanie. La solution que $\mathrm{j}$ 'ai proposée est «La hotte de l'Iscariote», en raison des connotations de traîtrise et de religion (ces enfants sont endoctrinés dans un pensionnat religieux) (p. 217).

Au cours de la «drôle de guerre» de 1940, que Claus relate avec une verve grotesque, un officier belge meurt écrasé sous un pont «pijp in de mond en de broek vol stront» (p. 307); la traduction littérale est «pipe à la bouche et la culotte pleine de merde», mais j'ai préféré garder le rythme et l'assonance rappelant ceux d'un dicton : «la pipe au bec et le cul pas sec» (p. 245).

Encore un passage délicat du Chagrin des Belges: Louis pendant la guerre, en vacances forcées chez des paysans allemands, leur récite à la veillée quelques vers du grand poète flamand Guido Gezelle. Comme on lui demande de répéter, agacé sans doute, il en fait une parodie instantanée. J'ai donc été obligé d'inventer un extrait de poème ressemblant vaguement à une traduction de Gezelle, puis je l'ai parodié dans le registre cru qui convenait.

Ik heb vanavond: "O, krinkelende winkelende waterding met uw zwarte kabotseken aan," enz. gereciteerd. Ik moest het nog eens doen en zei: " $O$, stinkende, winkelende paterdingsschart er uw rokske maar aan.» Niemand heeft het verschil gemerkt.

Ce soir, je leur ai récité : «O murmurante chuchotante source douce avec tes reflets noirs moirés», etc. On m'a demandé de le répéter et j'ai dit : «O purulente et chiante soupe aux choux avec tes reflets merdorés.» Personne n'a remarqué la différence.

Dans le clin d'œil complice lancé au lecteur, l'auteur de l'original se réfêre souvent à une réalité culturelle inconnue du lecteur de la traduction. Ceci fait que même si la traduction littérale convenait en cet endroit, une partie de l'humour serait perdu si on ne faisait pas, plutôt qu'une traduction, une adaptation faisant usage d'une référence bien connue dans la langue d'arrivée. Dans une des pièces de Witkiewicz, intitulée Tumeur Cervykal ${ }^{13}$, une mère de 7 enfants nommée Libidina (en polonais Rozhulantyna), berce son petit dernier sur une parodie de comptine polonaise qui parle de chatons et du papa. La comptine déraille dans le grotesque et le papa s'en va voir les hétaïres. Ceci est devenu en français:

\footnotetext{
Une poule sur un mur

Qui picote du pain dur

Picoti-picota

Lève la queue et saute en bas

La poule a piqué papa

Qui en est resté baba

Papa aime les piqûres

C'est un pourceau d'Épicure

Picuri-picura

Lève la queue et saute en bas.
} 
Papa a dit: Moi d'abord

C'est toi la poule aux cufs d'or

Mais c'est moi le picador

Picadi-picada

Lève la... (p. 47)

L'un des éléments humoristiques les plus frappants des pièces de Witkiewicz est sa façon d'affubler les personnages de noms aux consonances grotesques. Il arrive qu'il leur donne un nom qu'on peut conserver en français sans modification, ou presque, comme dans Gyubal Velleitar Dona Scabrosa Macabrescu, Dona Lubrica ou le marquis Simptoma da Fibroma. Parfois cependant une adaptation est nécessaire pour répondre à l'esprit ludique et gouailleur de Witkiewicz. Dans son théâtre, en effet, il est important de préserver une distance à l'égard des personnages et de leurs paroles et actes: il faut faire en sorte que soit maintenue la rupture voulue avec le réalisme, qui implique la négation de l'illusion théâtrale. Dès le début de la lecture d'une pièce, c'est-à-dire, dès la liste des personnages avec leur description, le lecteur et futur spectateur doit être plongé dans cette atmosphère qui tient du cirque et du cabaret. C'est ainsi que, avant même d'aborder Gyubal, on rencontrera les noms de Cochonnette Macabrescu, 10 ans, nymphette, du Dr Fornickmann, savant sadique, de Lydia Bellemiche, couturière jolie mais vulgaire, du baron Oskar von Mattrack, chef de la police, du Père Pungent, Grand Contife de l'Ordre infidèle des pneumatiques déchaussés, etc... ${ }^{14}$

On le voit, le traducteur ne s'ennuie pas toujours devant sa page blanche, mais il lui arrive de rester penché dessus, changé en statue de la perplexité, lorsque son auteur qu'il aime et qu'il admire, bien entendu - se laisse par trop aller à ses coupables penchants pour les jeux de mots ${ }^{15}$. Reprenons Nye et Faust et le narrateur Wagner qui s'aperçoit que, par une sorte de peur superstitieuse, il a évité de citer le nom de Faust pendant plusieurs chapitres :

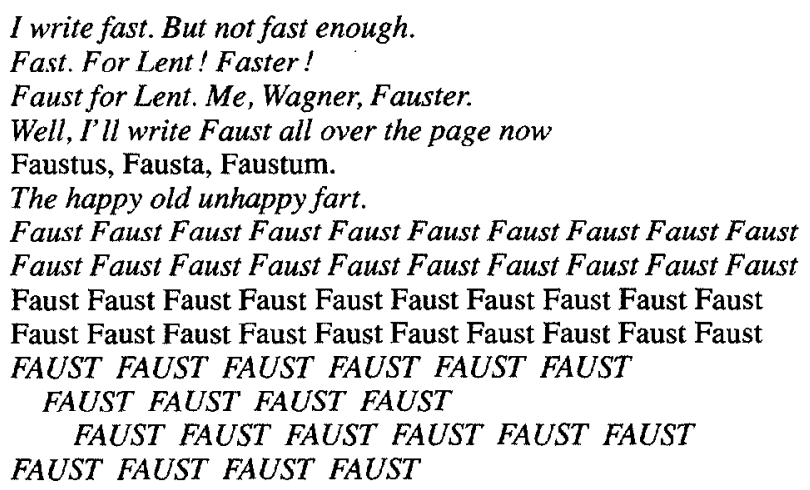

Go back if you like and stick them all in for yourself. (p. 39)

Estimant que Nye, en jouant sur Faust et fast (le jeûne de Carême), sur Faust et fosterchild (l'enfant adoptif qu'est Wagner), se payait ma tête autant que celle des autres lecteurs, je me suis senti autorisé à écrire n'importe quoi, ou presque : 
J'écris vite. Mais pas assez vite. Pas assez bien?

Est-ce que je subis son influence néfaste?

Néfaste Faust. Né faste? Né Faust.

Va pour les fastes de l'écriture.

Écrivons faste. Je vais écrire Faust sur toute la page.

$\cdots$

Retournez en arrière si vous avez envie et mettez-les où vous voulez.

(p. 61)

Une re-création, la traduction? Peut-être, mais avant tout une récréation.

Notes

1. Habitude, genre d'existence.

2. Trou.

3. Blond.

4. Feu.

5. Monnaie russe valant un douzième de florin.

6. Boisson fermentée des gens du peuple.

7. Bons conseils.

8. Robert Nye (1980) : Faust, London, Hamish Hamilton, p. 9.

9. Robert Nye (1986) : Faust, Paris, Julliard-L'Âge d'Homme, p. 26.

10. Marian Pankowski (1981): Nasz Julo Czerwony i Siedem innych sztuk, Londres, Oficyna Poetow i Malarzy, p. 133. Marian Pankowski (1978): Théâtre complet, vol. II, Lausanne, L'Âge d'Homme, p. 145.

11. J'avoue que je me suis aussi amusé ici à citer une autre parodie, celle du Cid par un autre poète un peu oublié. Paul Fourest, auteur de la Négresse blonde et du Géranium ovipare : Chimène, malgré sa rage et sa douleur, y admet finalement: «Qu'il est joli garçon, l'assassin de papa !»

12. Hugo Claus (1983): Het verdriet van België, Amsterdam, De Bezige Bij.Hugo Claus (1985) : le Chagrin des Belges, Paris, Juliard.

13. S.I. Witkiewicz (1985): Dziela wybrane, vol. IV, Warsawa, P.I.W.

14. Polonais : ibid., p. 588 sq. Français : ibid., vol. III, 1971, p. 49 sq.

15. C'est pourquoi j'aimerais proposer que les éditeurs payent leurs traducteurs à l'heure plutôt qu'à la ligne ; mais je crains que cette revendication candide ne soit pas prise en considération. 